\title{
Limiting Light Escape Angle in Silicon Photovoltaics: Ideal and Realistic Cells
}

\author{
Emily D. Kosten, Bonna K. Newman, Member, IEEE, John V. Lloyd, \\ Albert Polman, and Harry A. Atwater, Member, IEEE
}

\begin{abstract}
Restricting the light escape angle within a solar cell significantly enhances light trapping, resulting in potentially higher efficiency in thinner cells. Using an improved detailed balance model for silicon and neglecting diffuse light, we calculate an efficiency gain of $3 \%$ abs for an ideal Si cell of $3-\mu \mathrm{m}$ thickness and the escape angle restricted to $2.767^{\circ}$ under AM1.5 direct illumination. Applying the model to current high-efficiency cell technologies, we find that a heterojunction-type device with better surface and contact passivation is better suited to escape angle restriction than a homojunction type device. In these more realistic cell models, we also find that there is little benefit gained by restricting the escape angle to less than $10^{\circ}$. The benefits of combining moderate escape angle restriction with low to moderate concentration offers further efficiency gains. Finally, we consider two potential structures for escape angle restriction: a narrowband graded index optical multilayer and a broadband ray optical structure. The broadband structure, which provides greater angle restriction, allows for higher efficiencies and much thinner cells than the narrowband structure.
\end{abstract}

Index Terms-Nanostructures, photovoltaic cells, silicon.

\section{INTRODUCTION}

$\mathbf{S}$ ILICON solar cells are currently the dominant terrestrial photovoltaic technology due to material abundance and relatively low-cost manufacturing processes. Recently, a new performance record of $25.6 \%$ was achieved [1]. One approach to increasing efficiency is restricting the angles at which light may escape the cells with an external optic. Restricting the escape angle reduces the escape cone inside the cell, leading to two potential improvements: enhanced light trapping and increased photon recycling [2]-[4]. In materials with significant

Manuscript received June 29, 2014; revised September 11, 2014; accepted September 17, 2014. Date of publication October 22, 2014; date of current version December 18, 2014. The work of E. D. Kosten and H. A. Atwater was supported by the Light-Matter Interactions Energy Frontier Research Center: an EFRC program of the Office of Science, United States Department of Energy, under Grant DE-SC0001293. The work of E. D. Kosten was supported by the Resnick Sustainability Institute Graduate Fellowship. The work of J. V. Lloyd was supported by the DOW Chemical Company. Work at the Center for Nanophotonics at AMOLF is part of the research program of FOM, which is financially supported by NWO. It is also supported by the European Research Council. E. D. Kosten and B. K. Newman contributed equally to this work.

E. D. Kosten, J. V. Lloyd, and H. A. Atwater are with the Thomas J. Watson Laboratory of Applied Physics, California Institute of Technology, Pasadena, CA91125 USA (e-mail: haa@caltech.edu; jvlloyd@caltech.edu; haa@caltech. edu).

B. K. Newman was with the Center for Nanophotonics, FOM Institute AMOLF, 1098 XG Amsterdam, The Netherlands. She is now with ECN Solar Energy, 1755 ZG Petten, The Netherlands (e-mail: bonna.newman@gmail. com).

A. Polman is with the Center for Nanophotonics, FOM Institute AMOLF, 1098 XG Amsterdam, The Netherlands (e-mail: polman@amolf.nl).

Color versions of one or more of the figures in this paper are available online at http://ieeexplore.ieee.org.

Digital Object Identifier 10.1109/JPHOTOV.2014.2360566 radiative recombination such as GaAs, the photon recycling effect is significant [5]-[7]. However, in low radiative efficiency materials, like silicon, the light-trapping effect is more dominant, as light near the band edge is more completely absorbed [2], [3]. Limiting the light escape angle significantly enhances the light-trapping mechanisms already utilized in current flatplate silicon cells, allowing for excellent light absorption in a very thin cell and reduced materials usage.

While the limiting efficiencies for silicon cells under angle restriction were initially calculated by Campbell and Green in 1986 [3], we recalculate these efficiencies to include models for free carrier absorption [8], improved Auger parameterizations [9], and bandgap narrowing [10] that have been developed in the intervening decades. Using this model, we calculated a gain of $3 \%$ abs efficiency increase for an Auger-limited cell with maximum angle restriction $\left(2.767^{\circ}\right)$ and considering only the direct portion of the spectrum. We further develop the model to include surface and Shockley-Reed-Hall (SRH) recombination, allowing us to simulate the performance of current heterojunction and homojunction technologies [11]-[13]. We find that heterojuntion cells should be more ideal for angle restriction due to the lower surface recombination and the potential for thinner cells. We also find that excellent surface recombination and limited parasitic losses in inactive layers are crucial for significant efficiency improvements with angle restriction, while bulk lifetime has less of an impact.

When the escape angle is limited, the angles at which light can enter the cell are also limited, as a consequence of optical reciprocity. Thereby, escape angle restriction will also limit the portion of the diffuse spectrum that can be used by the device and the practical impact of escape angle restriction will depend upon the amount of diffuse light in a given location. However, for a concentrator system, the light is limited mostly to the direct portion of the spectrum as well. In these cases, we find that the gains from escape angle restriction are more clear and that combining moderate angle restriction with moderate concentration can offer more flexibility in system design and higher efficiencies.

There has been significant previous work on various designs for achieving angle restriction in silicon cells [3], [14]-[17]. However, most of these have focused on increasing short-circuit currents in very thin idealized cells. In contrast, we explore the effects of both narrowband rugate structures and broadband ray optical structures for angle restriction over a wide range of cell thicknesses and for both ideal and realistic cells. We find that broadband ray optical angle restrictors allow for larger efficiency enhancements than their narrowband counterparts, 
owing to the narrower angle restriction provided over a broader range of wavelengths when neglecting diffuse irradiance. With a broadband structure, efficiency increases up to $0.8 \%$ abs for heterojunction type cells and $1.5 \%$ abs for idealized cells are predicted for optimal cell thicknesses.

\section{EFFECTS OF ANGLE RESTRICTION IN IDEAL AND REAlistic Silicon CELlS}

To model the effects of angle restriction, we use a detailed balance approach, assuming intrinsic silicon, with uniform carrier density throughout the absorber layer, and excellent carrier collection [18]. The net current $J(V)$ is given by

$$
J(V)=J_{L}(V)-R_{A}(V) W-R_{\mathrm{RR}}(V)-J_{\mathrm{SRH}}-J_{\mathrm{SRV}} .
$$

Here, $J_{L}(V)$ is the light-generated current is given by

$$
J_{L}(V)=C_{f} \int_{0}^{\infty} a(E, V) S(E) d E
$$

where $S(E)$ is the AM 1.5 direct solar spectrum, and $C_{f}$ is the external concentration factor, with voltage dependence owing to the free carrier absorption. The band-to-band absorptivity $a(E, V)$ is

$$
a(E, V)=\frac{\alpha(E)}{\alpha(E)+\alpha^{\prime}(E, V)+\frac{\sin ^{2}\left(\theta_{e}\right)}{4 n_{r}^{2} W}}
$$

where $\alpha(E)$ and $\alpha^{\prime}(E, V)$ are the band-to-band and parasitic absorption coefficients, respectively, and $\theta_{e}$ is the angle defining the escape cone [2], [3], [19]. Thus, as the escape angle, $\theta_{e}$, is reduced, $a(E, V)$ increases, as the escape cone for light within the solar cell narrows.

In second term of (1), the quantity $R_{A}(V)$ is Auger recombination as a function of the electron and hole concentrations based on the recent parameterization determined by Richter et al. [8], [9], [18]. As Auger recombination is a bulk process, this term scales with the cell thickness, $W$. Electron and hole concentrations are determined from the assumed doping, the neutrality condition, the cell voltage, and the law of mass action [20]. Band gap narrowing is also included this term with intrinsic carrier concentration, $n_{i, 0}$ of $8.28 \times 10^{9} \mathrm{~cm}^{-3}$ [8], [10], [21], [22] at $T=25^{\circ} \mathrm{C}$.

The third term in (1), $R_{R R}(V)$, accounts for current losses due to radiative recombination processes. This is modeled as black-body type radiative emission at each photon energy $E$ and applied voltage $V$ and allows us to include angle restriction as follows:

$$
\begin{aligned}
R_{\mathrm{RR}}(V)= & \int_{0}^{\infty} \frac{2}{h^{3} c^{2}} \frac{E^{2}}{e^{(E-q V) / k T}-1} \\
& \times\left(\int_{\Omega_{c}} a(E) \cos (\theta) d \Omega+\pi n_{r}^{2} a^{\prime}(E)\right) d E
\end{aligned}
$$

where $h$ is Planck's constant, $c$ is the speed of light, $q$ is electron charge, and $k$ is Boltzmann's constant. The first term accounts for the radiative emission from the material with absorptivity $a(E)$ in the solid angle $\Omega_{c}$. For a planar emission, with escape angle restriction of $\theta_{e}$, the integral over $\Omega_{c}$ results in $\sin ^{2}\left(\theta_{e}\right)$.
TABLE I

MODEl PARAMETERS AND RESUlTS FOR IDEAL AND REALISTIC CELLS

\begin{tabular}{lccc}
\hline \hline & Ideal & heterojunction & homojunction \\
\hline Dopant(n) Density $\left(\mathrm{cm}^{-3}\right)$ & $1 \times 10^{11}$ & $1.6 \times 10^{15}$ & $1.6 \times 10^{15}$ \\
Back Reflectivity $(\%)$ & 100 & 98 & 95 \\
$\mathrm{SRV}\left(\mathrm{cm} \cdot \mathrm{s}^{-1}\right)$ & 0 & 2.87 & 11.64 \\
$\tau_{B}(\mathrm{~ms})$ & Infinite & 2.2 & 1.2 \\
\hline \multicolumn{4}{c}{ Modeled performance under AM1.5G illumination $\left(100 \mathrm{~mW} \cdot \mathrm{cm}^{-2}\right)$} \\
and no angle restriction. & \\
$\mathrm{V}_{\text {oc }}(\mathrm{mV})$ & 29.5 & 24.7 & 24.4 \\
Thickness $(\mu \mathrm{m})$ & 762 & 750 & 721 \\
\hline \multicolumn{1}{c}{ Modeled performance under AM1.5D illumination $\left(90 \mathrm{~mW} \cdot \mathrm{cm}^{-2}\right)$} & 180 \\
Max. Eff. $(\%)$ & 105 & 100 & 24.9 \\
Opt. Thickness $(\mu \mathrm{m})$ & 33.4 & 25.6 & 131 \\
\hline \hline
\end{tabular}

The second term is the parasitic absorptivity of spontaneously emitted light, $a^{\prime}(E, V)$, weighted by the square of the silicon refractive index, $n_{r}$, to account for the relative concentration of light within the solar cell [4], [18], [19], [23], [24]. The parasitic absorptivity is given by

$$
a^{\prime}(E, V)=4 W \alpha^{\prime}(E, V) \frac{\alpha(E)}{\alpha(E)+\alpha^{\prime}(E, V)+\frac{\sin ^{2}\left(\theta_{e}\right)}{4 n_{r}^{2} W}}
$$

where the amount of parasitic absorption is proportional to both the band-to-band emission, and the parasitic absorption coefficient, $\alpha^{\prime}(E)$ [4], [23], [25]. Absorption in nonideal back reflectors is included along with free carrier absorption in the parasitic absorption coefficient

$$
\alpha^{\prime}(E, V)=\alpha_{\mathrm{FCA}}(E, V)+\frac{1-R_{b}\left(1-a_{\mathrm{pass}}\right)}{4 W}
$$

where $\alpha_{\mathrm{FCA}}(E, V)$ is the free carrier absorption coefficient, $R_{b}$ is the back reflectivity, and $a_{\text {pass }}$ is the parasitic absorption per pass in inactive layers of the device [4], [23].

Finally, for more realistic cell models, we also include other types of nonradiative recombination

$$
\begin{aligned}
& J_{\mathrm{SRH}}=\frac{q W}{\tau_{B}} \frac{n p-n_{i \mathrm{eff}}^{2}}{p+n} \\
& J_{\mathrm{SRV}}=2 q S \frac{n p-n_{i \mathrm{eff}}^{2}}{p+n}
\end{aligned}
$$

where $\tau_{B}$ is the bulk lifetime associated with SRH processes, and $S$ is the effective surface recombination velocity (SRV) [26]. For both of these expressions, we assume a single SRV and SRH lifetime averaged over both carrier types, and, in the case of the SRV, both surfaces.

Using these relations, we calculate the maximum power under angle restriction as well as short-circuit current, $J_{\mathrm{SC}}$, and opencircuit voltage, $V_{\mathrm{OC}}$. Table I summarizes the parameters and results used in all three cell models: ideal, heterojunction, and homojunction. Because the amount and angular distribution of diffuse light will vary significantly depending on location, we calculate the cell performance with respect to only the AM1.5 direct spectrum [27], and efficiency is normalized to $90-\mathrm{mW} \cdot \mathrm{cm}^{-2}$ 


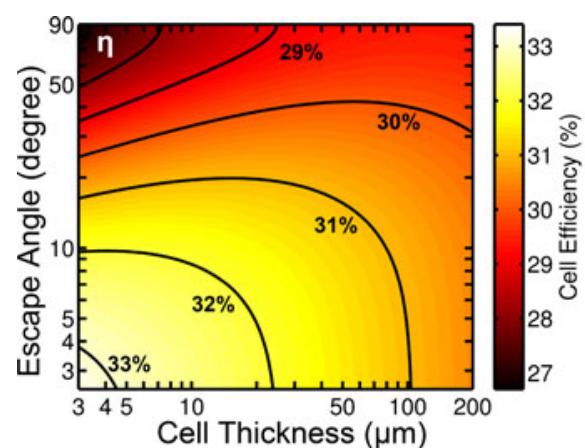

(a)

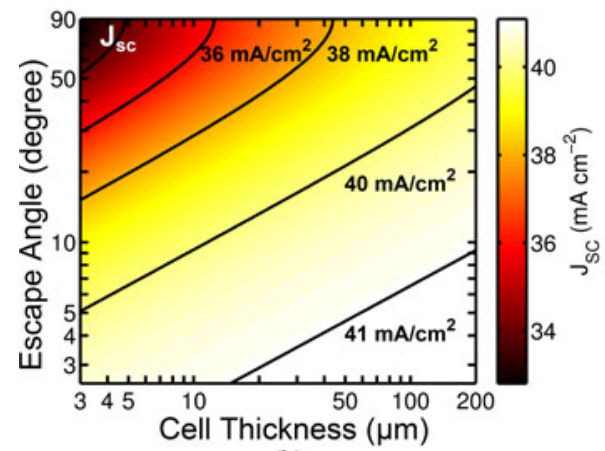

(b)

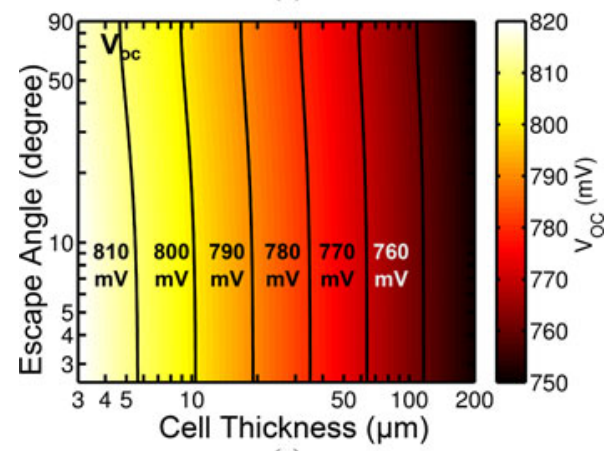

(c)

Fig. 1. Efficiency $\eta$, short-circuit current $J_{\mathrm{SC}}$, and open-circuit voltage $V_{\mathrm{OC}}$, for an ideal, Auger-limited silicon solar cell as a function of cell thickness and emission angle under AM1.5 direct spectrum illumination. Efficiency is reported with respect to $90 \mathrm{~mW} \cdot \mathrm{cm}^{-2}$ power flux. Increases in both $J_{\mathrm{SC}}$ and $V_{\mathrm{OC}}$ are observed by limiting escape angle and cell thickness. Narrow angle restriction and a very thin cell lead to the highest efficiency.

irradiance. We will discuss the potential impact of diffuse light below.

\section{A. Angle Restriction in Ideal Cells}

Fig. 1 shows the results for an n-type lowly-doped $\left(1 \times 10^{11}\right.$ $\mathrm{cm}^{-3}$ ) silicon substrate assuming a perfect back reflector and neglecting all nonradiative recombination other than Auger. The parameters are chosen to minimize Auger recombination and match the calculations from [9]. Highest efficiency, up to a $3 \%$ abs increase, is achieved for thinner cells with narrow angle restriction. While thinner cells show improved performance, we limit the minimum cell thickness to $3 \mu \mathrm{m}$, as the absorptivity expressions above apply only in the ray optics limit. Additionally, there is a limit to optimal thickness when more realistic losses are considered, as described in the next section. Most of the enhancement at a given thickness is due to improved light trapping and a corresponding increase in $J_{\mathrm{SC}}$ [see Fig. 1(b)] with less of an effect from photon recycling impacting $V_{\mathrm{OC}}$ [see Fig. 1(c)]. Increases in $V_{\mathrm{OC}}$ are mostly due to decreased impact from Auger recombination as thickness is reduced when surface recombination is neglected.

From the efficiency [see Fig. 1(a)], we see that optimal thickness decreases as angle restriction narrows. While narrow angle restriction and very thin cells lead to the highest efficiencies, for cells thicker than $50 \mu \mathrm{m}$, angle restriction narrower than $10^{\circ}$ has less of an impact on cell performance, suggesting that improvement for wafer-based silicon cells may be achievable, even with fewer restrictive escape angles.

\section{B. Angle Restriction in Realistic Cells}

While the limiting efficiency case is of theoretical interest, it is also important to consider the effects of angle restriction on current production-type silicon solar cell technologies: a-Si/c-Si heterojunctions with lower surface and contact recombination and homojunction devices. For this reason, we include additional losses due to bulk SRH and surface recombination in (1) as well as including imperfect back reflectors and parasitic losses within the cell.

Parameters for the ideal cell are chosen to match the assumptions from [8] for maximum performance of a Si solar cell. Cell thickness is chosen to match the literature values for similar record devices [11]-[13]. Back surface reflectance is assumed to be ideal for a pyramidal textured rear surface with $\mathrm{Ag}$ rear contacts: full coverage for the heterojunction-type cell and 3\% less in the homojunction-type cell to account for an all back contact design or local contact structure. For the heterojunction cell, we assume lowly doped n-type c-Si capped with $6 \mathrm{~nm}$ of a-Si on the front side and $9 \mathrm{~nm}$ of a-Si on the rear side [28], and parasitic absorption in these layers is calculated using the optical properties of a-Si [29]. Due to a lack of specific information of the optical properties of transparent conducting oxides (TCO) for this cell, we do not calculate these losses directly but assume all losses occur in the a-Si layers. For both cell types, we fit SRV and $\tau_{B}$ using the reported values of $V_{\mathrm{OC}}$ under the AM 1.5 global spectrum [11]-[13]. Modeled efficiencies, $V_{\mathrm{OC}}$, and optimal thickness for these reference cells, with and without angle restriction, are reported in Table I.

In Fig. 2, there is a contrast in the effects of angle restriction in heterojunctions versus homojunctions, despite similar reported efficiencies for these two cells [13]. To see this more clearly, we consider the $J_{\mathrm{SC}}$ and $V_{\mathrm{OC}}$ values plotted in Fig. 2. The absolute value of maximum $J_{\mathrm{SC}}$ under angle restriction is larger for the homojunction type device due to less parasitic losses in the inactive passivation layers, but the relative gain in $J_{\mathrm{SC}}$ is greater in the thinner heterojunction device architecture. The potential impact in $V_{\mathrm{OC}}$ is also greater in heterojunctions due to effectively lower Auger recombination (tied to thickness) and less impact from surface/contact recombination. For both cell types, at all thicknesses, most of the increase in efficiency occurs 


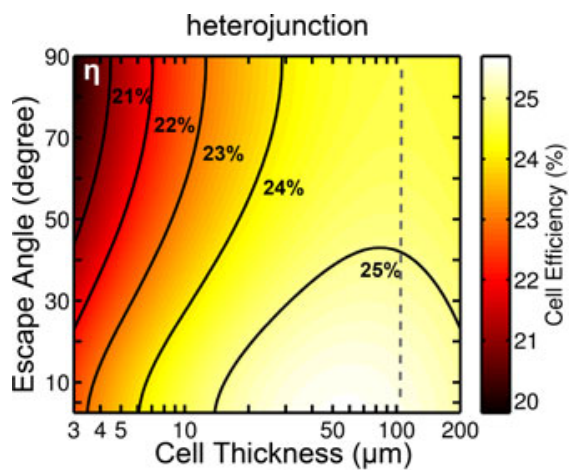

(a)

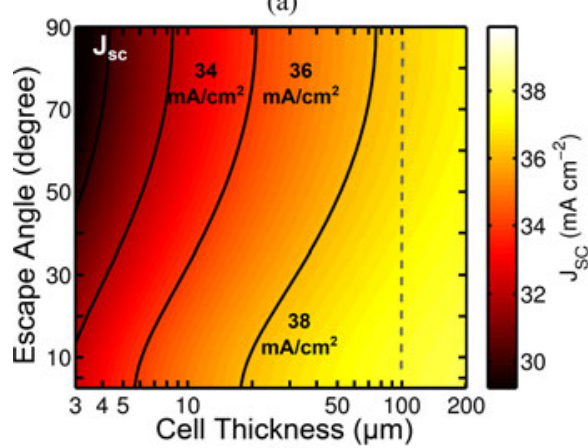

(c)

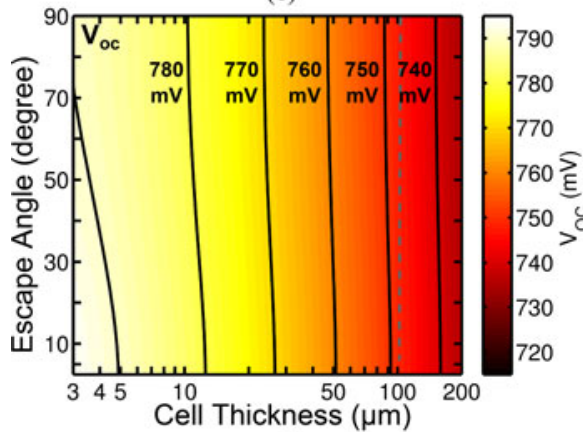

(e)

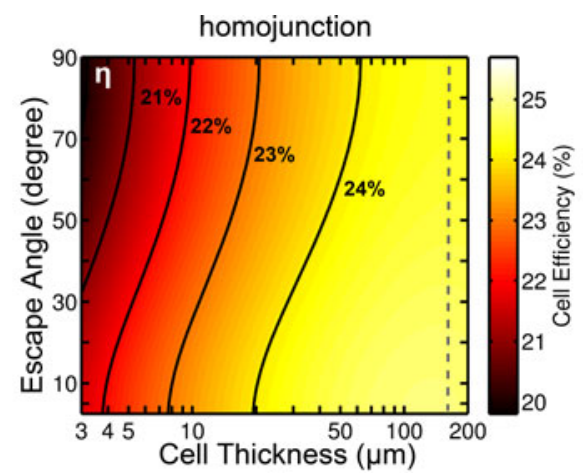

(b)

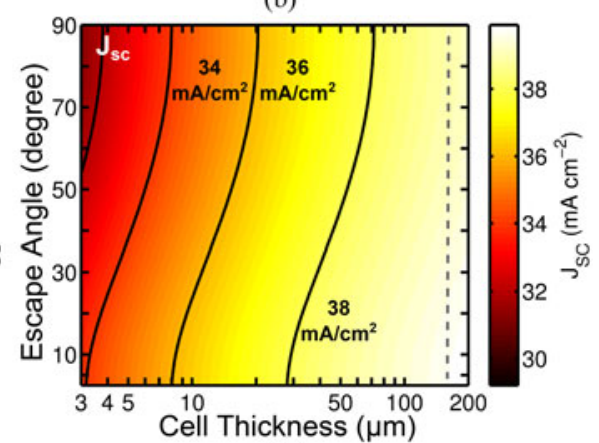

(d)

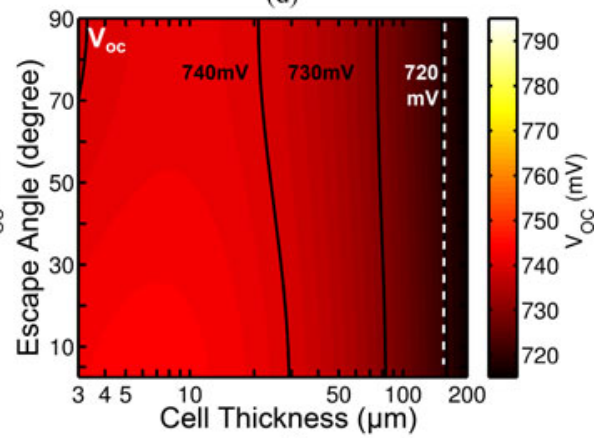

(f)

Fig. 2. Efficiency $\eta$, short-circuit current $J_{\mathrm{SC}}$, and open-circuit voltage $V_{\mathrm{OC}}$ as a function of cell thickness and escape angle for cells with surface recombination and SRH lifetimes mimicking heterojunction and homojunction type silicon cells. Vertical dashed lines represent the thickness of the reference cells. Heterojunction cells show greater efficiency improvements with angle restriction.

between $10^{\circ}$ and $70^{\circ}$ suggesting that maximum angle restriction may not be necessary or optimal.

Now, we use the complete model to further explore the relative impact surface/contact recombination, bulk recombination, and optical losses in realistic cells. In this discussion, we account for all optical losses due to imperfect reflectance and parasitic absorption in inactive layers as a single value of back reflectivity $R_{b}$. In Fig. 3(a) and (b), we plot the efficiency and efficiency increase under angle restriction as a function of back reflectivity, as well as examine the effect of a factor of two improvement in either SRV or bulk lifetime. While improvements in either back reflectivity, SRV, or lifetime lead to an overall efficiency enhancement, as shown in Fig. 3(a), SRV and back reflectivity lead to greater efficiency increase with angle restriction as shown in Fig. 3(b). As shown in Fig. 3(c), which gives the optimal cell thickness at $10^{\circ}$ angle restriction as a function of back reflectivity, improved SRV and back reflectivity lead to larger improvements with angle restriction as they allow for thinner optimal cells. In contrast, the optimal cell thickness under angle restriction is larger for longer lifetimes, as bulk recombination is reduced. Thus, improving SRV and back reflectivity are crucial to achieving thin high-efficiency silicon cells under angle restriction.

\section{Angle Restriction With External Concentration}

So far, we have considered the effect of escape angle restriction for enhanced light trapping in the solar cell. Next, we consider how external concentration can further enhance the performance of a Si device when used in combination with the same angle restriction geometry. An external concentrator will also limit the optical acceptance angle. Light is collected from a limited range of incoming angles and concentrated onto a smaller area with a broader angular spread. At the thermodynamic limit [30] of concentration by the factor $C_{f}$, the relationship between the angular spread of incoming light $\theta_{\text {in }}$ and the angular spread 


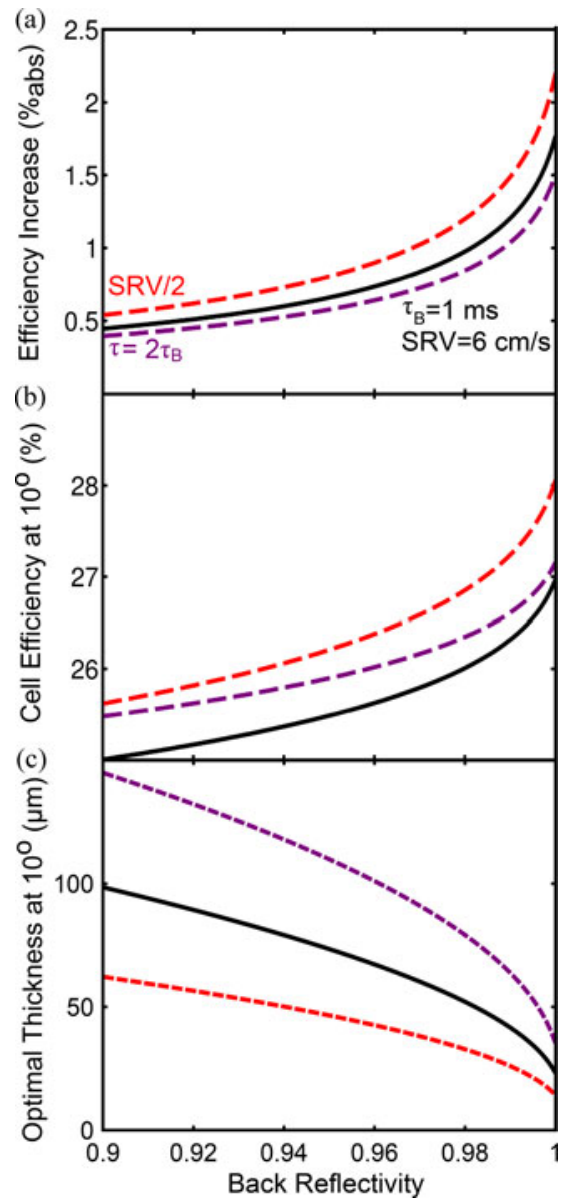

Fig. 3. (a) Cell efficiency, (b) efficiency increase, and (c) optimal thickness at $10^{\circ}$ restricted angle, as a function of back reflectivity for a heterojunction-type cell with SRH lifetime $\tau_{B}=1 \mathrm{~ms}$ and SRV $=6 \mathrm{~cm} \cdot \mathrm{s}^{-1}$ (black) and the same data for $\tau_{B}=2 \mathrm{~ms}$ (dashed purple) and SRV $=3 \mathrm{~cm} \cdot \mathrm{s}^{-1}$ (dashed red).

of light after concentration $\theta_{\text {out }}$ is

$$
\sin \left(\theta_{\text {out }}\right)=\sqrt{C_{f}} \sin \left(\theta_{\text {in }}\right) .
$$

For solar radiation with perfect tracking, the direct spectrum is incident from a limited set of angles spanned by the image of the sun on the earth's surface and the spread of the circumsolar radiation [27]. Due to reciprocity, the angular spread of light after concentration limits the narrowest possible solar cell acceptance angle where all incoming light is collected and, therefore, the minimum emission angle $\theta_{\text {out }}$.

In Fig. 4(a) and (b), we plot the efficiency and optimal thickness, respectively, of a heterojunction cell with angle restriction combined with various levels of concentration, neglecting heating or increased series resistance losses in the cells. Without concentration, efficiency can be improved up to $0.8 \%$ abs by narrowing the escape angle to $20^{\circ}$ consistent with Fig. 4(a). With concentration, the optimal thickness increases due to the increased carrier density and increased recombination rates. Conversely, and similar to the case with no concentration, we see that the optimal thickness decreases with increased angle restriction (assuming no heating). Thus, for a given concentration, higher efficiency could be reached with a thinner device.

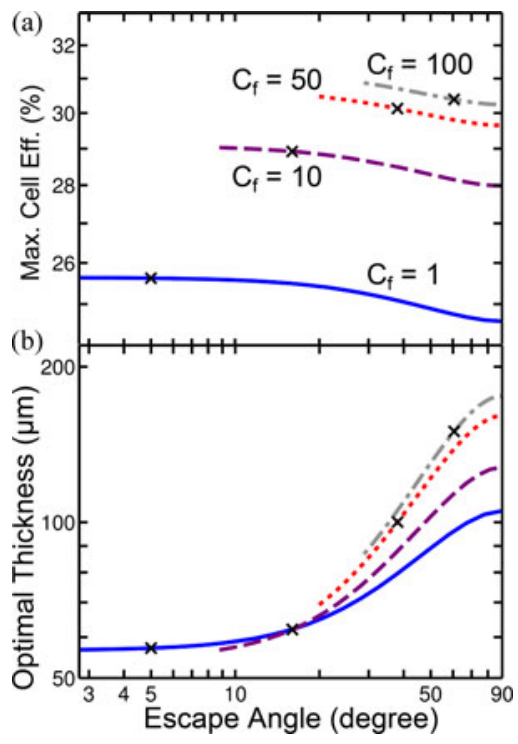

Fig. 4. (a) Maximum cell efficiency and (b) corresponding optimal cell thickness as a function of escape angle for an heterojunction-type cell with various external concentrations $C_{f}$, assuming ideal tracking precision indicated by the lines. Results for $5^{\circ}$ tracking precision are indicated by black "x."

As mentioned previously, an angle restriction scheme would likely require tracking to ensure the sun's image falls within the allowed angles for light to enter the cell. A typical low-precision tracking system for solar is on the order of $5^{\circ}$; performance limitations for such a tracker are also indicated in Fig. 4. With such a tracking system, using 50x concentration in combination with moderate angle restriction of $40^{\circ}$, a heterojunction device could achieve greater than $30 \%$ efficiency (with respect to the direct spectrum) with a 100- $\mu \mathrm{m}$ substrate. The tradeoff between thickness, concentration, and angle restriction offers a variety of options for attaining higher efficiency with a range of various module geometries.

\section{Impact of Diffuse Light}

By reciprocity and assuming conservation of entropy, any amount of angle restriction also limits the acceptance angle for absorption; a minimum of $10 \%$ of the ambient light in the case of the ASTM173 standards used here. For specific locations, the distribution of diffuse light will depend on scattering from molecules, aerosols, and clouds and in areas with high direct normal irradiance it will likely not be isotropically distributed. Thus, we have restricted the calculations to only considering the direct portion of the spectrum, even for cells with no angle restriction. All efficiency gains previously reported are only with respect to the normally incident portion of the spectrum regardless of angle restriction. To account for diffuse illumination, a specific model of the angular and spectral distribution of diffuse light would be necessary. To first order, however, we can evaluate the potential of angle restriction by examining peak power at two extremes: 1) a cell with no angle restriction under global illumination $\left(100 \mathrm{~mW} \cdot \mathrm{cm}^{-2}\right)$ and 2) the same cell with maximum angle restriction $\left(2.767^{\circ}\right)$ under direct illumination 
$\left(90 \mathrm{~mW} \cdot \mathrm{cm}^{-2}\right)$. The modeled efficiencies are summarized in Table I. For the ideal cell, we find that angle restriction could result in an overall gain of $0.59 \mathrm{~mW} \cdot \mathrm{cm}^{-2}$ (2.0\% relative). For the heterojunction and homojunction devices, we find a loss of $1.62 \mathrm{~mW} \cdot \mathrm{cm}^{-2}(-6.6 \%$ relative $)$ and $2.0 \mathrm{~mW} \cdot \mathrm{cm}^{-2}(-8.2 \%$ relative), respectively. This suggests that only in a device with minimal parasitic loss and nearly ideal material characteristics would gain from angle restriction overcome the loss of current from diffuse light. However, this assumes narrow angle restriction across the full spectrum. In practice, short wavelength light is strongly scattered in the atmosphere and dominates the diffuse light spectrum, while angle restriction is primarily beneficial as light trapping for longer wavelengths. A narrowband angle restrictor, optimized for light trapping at longer wavelengths, near the band edge, and transmission of high angle (diffuse) light at short wavelengths, may allow for a performance enhancement in more realistic cells with more significant parasitic losses. The design and performance of such a narrowband angle restrictor is discussed further in the next section.

\section{ANGLE RESTRICTOR DESIGNS}

In all the preceding calculations, we have assumed an ideal angle restrictor: lossless, with either $100 \%$ reflection or transmission, and no wavelength dependence. There has been significant prior work focused on designing such structures for both crystalline and amorphous silicon cells [3], [14]-[17]. However, most of this work has focused on structures that limit the escape angle only over a narrow wavelength range for very thin, idealized solar cells or has been more conceptual. Here, we consider both narrowband and broadband angle restrictors and analyze the effects of these structures on the performance of both the idealized and heterojunction silicon cells over a range of thicknesses.

\section{A. Narrowband Angle Restrictor}

To achieve narrowband angle restriction, we consider a multilayer structure with alternating high and low refractive index. While the design considered here is more sophisticated, the angle restriction effect may be understood by considering the Bragg condition for reflection from a periodic stack with alternating high and low index layers

$$
\cos \theta_{m}=\frac{m \lambda}{2 \Gamma}
$$

where $\theta_{m}$ is the angle of maximum reflectivity, $\lambda$ is the wavelength, $\Gamma$ is the period of the multilayer, and $m$ is an integer [31]. Thus, for shorter wavelengths, maximum reflection occurs away from normal incidence achieving the desired angle restriction effect. To avoid undesirable second-order reflecting bands, and other normal incidence reflections, we utilize a rugate structure, with a gradual variation of refractive index [15], [16], [32], [33]. We assume the cell is placed under glass $(n=1.5)$, as is common in a module, and that gradual index variation between 1.5 and 2.5 may be achieved with $\mathrm{SiO}_{2} / \mathrm{TiO}_{2}$ codeposition [34][36]. We also assume that the rugate structure is deposited in place of an antireflective (AR) coating between the glass and the

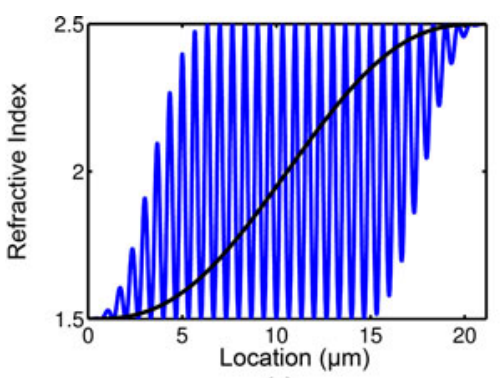

(a)

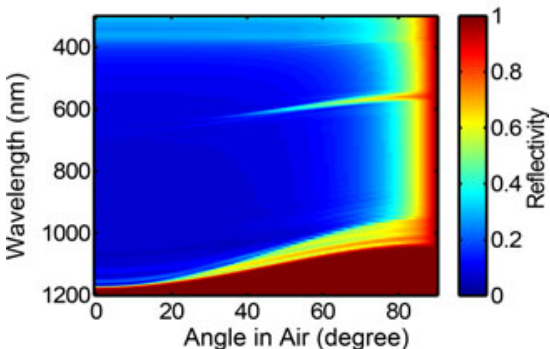

(b)

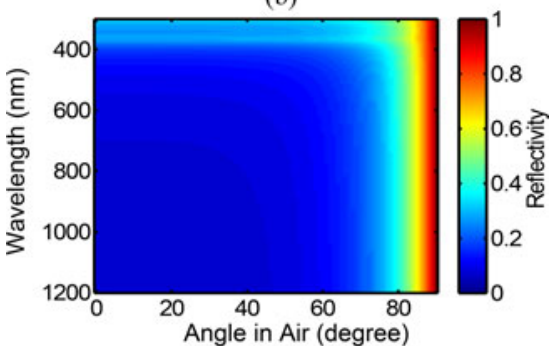

(c)

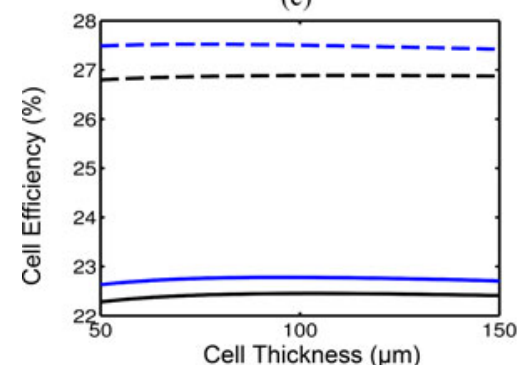

(d)

Fig. 5. (a) Refractive index profile for rugate angle restrictor design (blue) and quintic graded index AR coating for comparison (black). (b) Calculated reflectivity as a function of incident angle (relative to the surface normal) and wavelength for rugate angle restrictor design and (c) graded index AR coating. (d) Calculated efficiency as a function of cell thickness with rugate angle restrictor (blue) and graded index AR coating (black) for the heterojunction cell model (solid) and the ideal cell model (dashed).

solar cell. As a comparison case, we consider a graded index AR coating with a quintic-type refractive index profile deposited at the same interface and with the same range of refractive index [37]. The transfer-matrix method is used to calculate the performance of the thin film structures. Reflections off the overlying glass $(n=1.5)$ were included with a multipass model. Fig. 5(a) shows the refractive index profiles of the optimized rugate anglerestricting structure and the graded index coating.

The calculated reflectivity as a function of angle of incidence and wavelength for the two coatings is shown in Fig. 5(b) and (c). The reflectance at normal incidence is very similar for 
both cases. Thus, the rugate structure avoids additional optical losses. The rugate structure shows a sharp transition in reflectivity at a given angle, in the wavelength range $1050-1200 \mathrm{~nm}$. In this wavelength range, the light escape cone is thus limited, as desired.

To calculate the performance of a cell with the rugate anglerestricting coating described above, we replace the factors of $\sin ^{2}\left(\theta_{e}\right)$ in the model with a wavelength dependent angleaveraged transmission, determined from the reflectivity results shown in Fig. 5(b) and (c). As shown in Fig. 5(d), for an ideal cell, optimal thickness decreases from 110 to $80 \mu \mathrm{m}$. For the heterojunction cell, efficiency increases $0.3 \%$ abs with a $7-\mu \mathrm{m}$ decrease in optimal thickness. As the angle restriction is relatively narrowband, the angle restrictor will still capture a majority of the diffuse light in the short wavelength range and any gains in efficiency for the direct portion of the spectrum would likely improve the overall performance of the system under realistic conditions.

\section{B. Broadband Angle Restrictor}

The results above suggest that narrower angle restriction over a broader wavelength range will be required to achieve the efficiency increases calculated for the ideal angle restrictor. To explore this further, we examined a broadband ray-optical angle restrictor, which utilizes an array of hexagonal solid compound parabolic concentrator (CPC) structures as shown in Fig. 6(a) [30]. In this design, similar to that proposed by Green, the CPC structure utilizes total internal reflection to direct light near normal incidence to the output aperture where it enters the cell [3], [14]. Except for the area under the output apertures of the CPCs, the top surface of the cell is coated with a metallic reflector, such that light inside the cell can only escape through the output apertures and light trapping is enhanced.

As in the previous section, we assume that the CPC array and solar cell is under the glass with index of 1.5. We also assume that the solid CPC structures have constant refractive index of 1.5, and that the graded-index AR coating presented in Fig. 5 is deposited between the CPC output apertures and the silicon cell below. Ray tracing simulations, were used to calculate the reflectivity as a function of incident angle for the wavelength range of interest, and representative results at $1000 \mathrm{~nm}$ are shown in Fig. 6(b). At other wavelengths, the reflectivity is similar with the small wavelength dependence due to the AR coating between the CPC array and the silicon below. We note that achieving narrow angle restriction with good transmission requires including a conical section at the bottom of the $\mathrm{CPC}$ to narrow the range of output angles from the structure and avoid rejected skew rays [30]. As shown in Fig. 6(b), the conical section leads to a higher aspect ratio structure. Ideally, however, the spacing between the reflector holes would be no larger than the carrier diffusion length (typically 2-3 mm for n-type solar $\mathrm{Si}$ ); therefore, the CPC structure would be on the order of a few to several millimeter tall.

Similar to the previous section, we incorporate the ray tracing results into the detailed balance model. We also include the losses from the nonideal reflector between the CPC apertures.

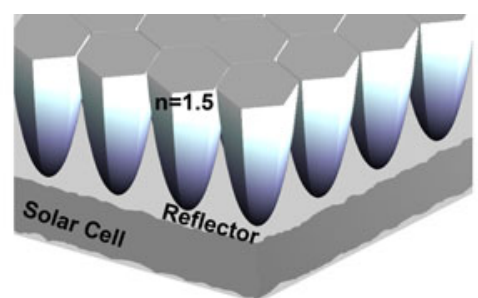

(a)

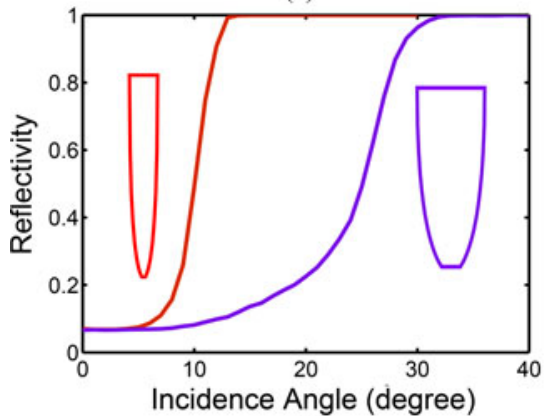

(b)

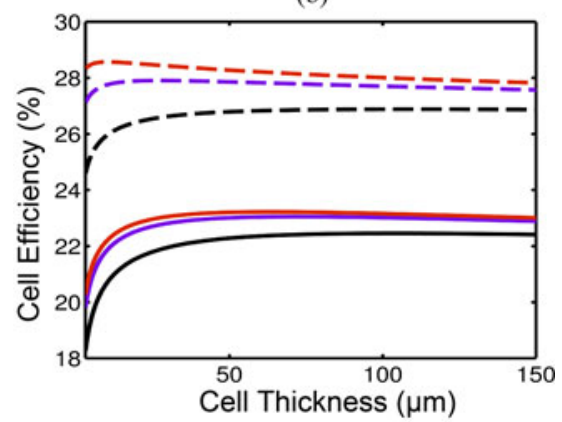

(c)

Fig. 6. (a) CPC-based ray-optical angle restrictor schematic. (b) Reflectivity as a function of incident angle from ray-tracing simulations at 1000-nm wavelength for two CPC shapes (shown in insets) with effective angle restriction of $10^{\circ}$ (red) and $25^{\circ}$ (purple), respectively. (c) Calculated efficiency as a function of thickness for the heterojunction cell model (solid lines) and ideal cell model (dashed lines) are shown for graded index AR coating (black) and the CPC arrays [red and purple according to (b)]

Thus, the fraction of the light that escapes $P_{\text {esc }}$ replacing $\sin ^{2}\left(\theta_{e}\right)$ is now expressed as

$$
P_{\mathrm{esc}}=\bar{T}(\lambda)+A_{r}\left(1-R_{t}\right) n_{g}^{2}
$$

where $\bar{T}$ is the angle-averaged transmission determined from ray tracing, $A_{r}$ is the fraction of the top surface area covered by the reflector, and $R_{t}$ is the reflectivity of reflector, which covers the top surface of the cell between the CPC output apertures, which is assumed to be $98 \%$ in this case. $n_{g}$ is the refractive index of the material between the solar cell and reflector, which is assumed to be 1.5 .

Using the above expression, we calculate the efficiency as a function of cell thickness for both the ideal and heterojunction cell models for two different CPC designs. The narrower CPC shape is defined by a $5^{\circ}$ acceptance angle with a $57^{\circ}$ output angle, with an effective angle restriction of $10^{\circ}$. The broader CPC geometry has a $15^{\circ}$ acceptance angle with an $83^{\circ}$ output angle, with an effective $25^{\circ}$ angle restriction. Refraction at the glass-air interface leads to the larger effective acceptance angle [30]. As 
shown in Fig. 6(c), for the smaller escape angles, the efficiency improvements suggested by Figs. 1 and 2 are realizable. For the heterojunction structure, $0.8 \%$ abs efficiency improvement is found for the CPC structure with $10^{\circ}$ escape angle, with the optimal thickness decreasing substantially to $62 \mu \mathrm{m}$. For the $\mathrm{CPC}$ structure with a larger, $25^{\circ}$, escape angle, an efficiency enhancement of $0.6 \%$ abs is observed. Such a structure would also allow for cheaper, less-accurate tracking, and more utilization of diffuse light. For the ideal cell, Fig. 6(c) shows the effects of the CPC geometry are larger, with optimal thicknesses as thin as $10 \mu \mathrm{m}$ and efficiency increases of $1.5 \%$ abs. Thus, significant efficiency benefits for silicon solar cells are possible with realistic angle-restricting optical structures.

\section{CONCLUSION}

Restricting the light escape angle with an external optic has potential to further improve the performance of silicon solar cells by improving light trapping, allowing for thinner, more efficient cells in a flat plate geometry. Considering only the normally incident direct spectrum, and using a detailed balance approach, we have reevaluated the ideal, Auger-limited case and found that efficiency increases of up to $3 \%$ abs may be expected with very thin cells and narrow angle restriction. Considering the performance characteristics of record heterojunction and homojunction cells, we have found that the efficiency benefits of angle restriction are most significant in the heterojunction case, where $1.0 \%$ abs efficiency increases under AM1.5 direct irradiance are expected for cells that are half as thick as current optimum cells. Unlike the idealized case, heterojunction and homojunction devices with surface and bulk recombination, and including parasitic optical losses, we find that limiting the escape angle more narrowly than $10^{\circ}$ has minimal additional benefit. Angle restriction may also be used in concert with low to moderate external concentration, for addition efficiency enhancements. Loss of high angle, diffuse light requires excellent surface passivation and limited parasitic losses in inactive layers and reflections. Minimizing these current losses are crucial to achieving the maximal performance benefits with angle restriction. Finally, we have considered both narrowband rugate-based and broadband ray-optical angle restrictor designs. With the rugate structure, narrow escape angles are limited to a specific bandwidth (in this case 1050-1200 nm), and efficiency benefits are modest for both the heterojunction and ideal structures, with small changes in the optical thickness, but potentially improved use of diffuse light. In contrast, broadband angle restrictors can result in narrower escape angles, and show significant efficiency benefits and reductions in the optimal cell thickness, but might remain limited due to the loss of diffuse light. Based on these calculations, recent results with combined interdigitated back contact and heterojunction with intrinsic layer technology [1], and recently demonstrated technologies for micro-CPC-based structures [7], [38] and high-quality thin c-Si substrates [39][42] make this concept particularly relevant today. We envision broadband angle restriction with a CPC-based ray optical structure used in either a flat plate geometry or with low to moderate external concentration to demonstrate enhanced performance of crystalline silicon solar cells as they approach fundamental materials limitations.

\section{ACKNOWLEDGMENT}

The authors would like to thank M. Sheldon, H. Emmer, and W. Sinke for insightful discussions and advice on the manuscript.

\section{REFERENCES}

[1] (2014, Apr. 10). "Panasonic HIT solar cell: $25.6 \%$ efficiency world record," [Online]. Available: http://eu-solar.panasonic.net/en.

[2] E. Yablonovitch, "Statistical ray optics," J. Opt. Soc. Amer, vol. 72, no. 7, pp. 899-907, 1982.

[3] P. Campbell and M. Green, "The limiting efficiency of silicon solar cells under concentrated sunlight," IEEE Trans. Electron. Devices, vol. ED-33, no. 2, pp. 234-239, Feb. 1986.

[4] A. Martí, J. Balenzategui, and R. Reyna, "Photon recycling and Shockely's diode equation," J. Appl. Phys., vol. 82, no. 8, pp. 4067-4075, 1997.

[5] A. Braun, E. Katz, D. Feuermann, B. Kayes, and J. M. Gordon, "Photovoltaic performance enhancement by external recycling of photon emission," Energy Environ. Sci., vol. 6, pp. 1499-1503, 2013.

[6] O. Höhn, T. Kraus, G. Bauhuis, U. T. Schwarz, and B. Bläsi, "Maximal power output by solar cells with angular confinement," Opt. Exp., vol. 22, no. S3, pp. A715-A722, May 2014.

[7] E. Kosten, J. Atwater, J. Parsons, A. Polman, and H. Atwater, "Highly efficient GaAs solar cells by limiting light emission angle," Light: Sci. Appl., vol. 2, art. no. e45, 2013.

[8] A. Richter, M. Hermle, and S. Glunz, "Reassessment of the limiting efficiency for crystalline silicon solar cells," IEEE J. Photovoltaics, vol. 3, no. 4, pp. 1184-1191, Oct. 2013.

[9] A. Richter, S. Glunz, F. Werner, J. Schmidt, and A. Cuevas, "Improved quantitative description of Auger recombination in crystalline silicon," Phys. Rev. B, vol. 86, art. no. 165202, Oct. 2012.

[10] A. Schenk, "Finite-temperature full random-phase approximation model of band gap narrowing for silicon device simulation," J. Appl. Phys., vol. 84, no. 7, pp. 3684-3695, 1998.

[11] M. Taguchi, A. Yano, S. Tohoda, K. Matsuyama, Y. Nakamura, T. Nishiwaki, K. Fujita, and E. Maruyama, "24.7\% record efficiency HIT solar cell on thin silicon wafer," IEEE J. Photovoltaics, vol. 4, no. 1, pp. 96-99, Jan. 2014.

[12] P. Cousins, D. Smith, H.-C. Luan, J. Manning, T. Dennis, A. Waldhauer, K. Wilson, G. Harley, and W. Mulligan, "Generation 3: Improved performance at lower cost," in Proc. 35th IEEE Photovoltaic Spec. Conf., Jun. 2010, pp. 000 275-000 278.

[13] M. A. Green, K. Emery, Y. Hishikawa, W. Warta, and E. D. Dunlop. (2013). Solar cell efficiency tables (version 42). Prog. Photovoltaics. [Online]. vol. 25, no. 2, pp. 827-837. Available: http://dx.doi.org/10.1002/pip.2404

[14] A. Luque, "The confinement of light in solar cells," Solar Energy Mater., vol. 23, pp. 152-163, 1991.

[15] S. Fahr, C. Ulbrich, T. Kirchartz, U. Rau, C. Rockstuhl, and F. Lederer, "Rugate filter for light-trapping in solar cells," Opt. Exp., vol. 16, no. 13, pp. 9332-9343, 2008.

[16] C. Ulbrich, S. Fahr, J. Upping, M. Peters, T. Kirchartz, C. Rockstuhl, R. Wehrspohn, A. Gombert, F. Lederer, and U. Rau, "Directional selectivity and ultra-light-trapping in solar cells," Phys. Status Solidi A, vol. 205, no. 12 , pp. 2831-2843, 2008.

[17] P. Bermel, C. Luo, L. Zeng, L. C. Kimerling, and J. D. Joannopoulos. (2007, Dec.). Improving thin-film crystalline silicon solar cell efficiencies with photonic crystals. Opt. Exp.. [Online]. vol. 15, no. 25, pp. $16986-$ 17 000. Available: http://www.opticsexpress.org/abstract.cfm?URI=oe$15-25-16986$

[18] W. Shockley and H. Queisser, "Detailed balance limit of efficiency of p-n junction solar cells," J. Appl. Phys., vol. 32, no. 3, pp. 510-519, 1961.

[19] M. A. Green. (2008). Self-consistent optical parameters of intrinsic silicon at 300k including temperature coefficients. Sol. Energy Mater. Sol. Cells. [Online]. vol. 92, no. 11, pp. 1305-1310. Available: http://www. sciencedirect.com/science/article/pii/S0927024808002158

[20] S. Sze and K. Ng, Physics of Semiconductor Devices. New York, NY, USA: Wiley-Interscience, 1998. 
[21] P. P. Altermatt, A. Schenk, F. Geelhaar, and G. Heiser. (2003). Reassessment of the intrinsic carrier density in crystalline silicon in view of bandgap narrowing. J. Appl. Phys. [Online]. vol. 93, no. 3, pp. 1598-1604. Available: http://scitation.aip.org/content/aip/journal/jap/93/3/10.1063/1. 1529297

[22] A. Sproul and M. Green, "Intrinsic carrier concentration and minority carrier mobility of silicon from 77 to 300 k," J. Appl. Phys., vol. 73, no. 3, pp. 1214-1225, Feb. 1993.

[23] O. D. Miller, E. Yablonovitch, and S. R. Kurtz, "Strong internal and external luminescence as solar cells approach the Shockley-Queisser limit," IEEE J. Photovoltaics, vol. 2, no. 3, pp. 303-311, Jul. 2012.

[24] T. Tiedje, E. Yablonovitch, G. Cody, and B. Brooks, "Limiting efficiency of silicon solar cells," IEEE Trans. Electron Devices, vol. ED-31, no. 5, pp. 711-716, May 1984.

[25] I. Schnitzer, E. Yablonovitch, C. Caneau, and T. Gmitter, "Ultrahigh spontaneous emission quantum efficiency, $99.7 \%$ internally and $72 \%$ externally, from $\mathrm{AlGaAs} / \mathrm{GaAs} / \mathrm{AlGaAs}$ double heterostructures," Appl. Phys. Lett., vol. 62, no. 2, pp. 131-133, 1992.

[26] J. Nelson, The Physics of Solar Cells. London, U.K.: Imperial College Press, 2003.

[27] Standard Tables for Reference Solar Spectral Irradiances: Direct Normal and Hemispherical on $37^{\circ}$ Tilted Surface., ASTM Std. G173-03R12, 14th ed. West Conshocken, PA, USA: ASTM Int., 2012.

[28] Z. Holman, A. Descoeudres, L. Barraud, F. Fernandez, J. Seif, S. De Wolf, and C. Ballif, "Current losses at the front of silicon heterojunction solar cells," IEEE J. Photovoltaics, vol. 2, no. 1, pp. 7-15, Jan. 2012.

[29] E. Palik. (1998). Handbook of Optical Constants of Solids (ser. Handbook of Optical Constants of Solids) [Online]. vol. 4, Available: http:// books.google.nl/books?id $=$ rxuG1kXvSqgC

[30] W. Welford and R. Winston, High Collection Nonimaging Optics. New York, NY, USA: Academic, 1989.

[31] B. Saleh and M. Teich, Fundamentals of Photonics, 2nd Ed. New York, NY, USA: Wiley, 2007.

[32] B. G. Bovard, "Rugate filter theory: An overview," Appl. Opt., vol. 32, no. 28, pp. 5427-5442, 1993.

[33] W. H. Southwell, "Using apodization functions to reduce sidelobes in rugate filters," Appl. Opt., vol. 28, no. 23, pp. 5091-5094, 1989.

[34] W. J. Gunning, R. L. Hall, F. J. Woodberry, W. H. Southwell, and N. S. Gluck, "Codeposition of continuous composition rugate filters," Appl. Opt., vol. 28, no. 14, pp. 2945-2948, 1989.

[35] J.-S. Chen, S. Chao, J.-S. Kao, H. Niu, and C.-H. Chen, "Mixed films of $\mathrm{TiO}_{2}-\mathrm{SiO}_{2}$ deposited by double electron-beam coevaporation," Appl. Opt., vol. 35, no. 1, pp. 90-96, 1996.

[36] H. Demiryont, "Optical properties of $\mathrm{SiO}_{2}-\mathrm{TiO}_{2}$ composite films," Appl. Opt., vol. 24, no. 16, pp. 2647-2650, 1985.

[37] W. H. Southwell, "Gradient-index antireflection coatings," Opt. Lett., vol. 8, no. 11, pp. 584-586, 1983.

[38] J. H. Atwater, P. Spinelli, E. Kosten, J. Parsons, C. Van Lare, J. Van de Groep, J. Garcia de Abajo, A. Polman, and H. A. Atwater, "Microphotonic parabolic light directors fabricated by two-photon lithography," Appl. Phys. Lett., vol. 99, no. 15, art. no. 151113, 2011.

[39] K. V. Nieuwenhuysen, M. R. Payo, I. Kuzma-Filipek, J. V. Hoeymissen, G. Beaucarne, and J. Poortmans, "Epitaxially grown emitters for thin film silicon solar cells result in $16 \%$ efficiency," Thin Solid Films, vol. 518, no. 6, Suppl. 1, pp. S80-S82, 2010. sixth Int. Conf. Silicon Epitaxy Heterostructures. [Online]. Available: http://www.sciencedirect.com/science/article/pii/S0040609009017040

[40] P. Rosenits, F. Kopp, and S. Reber. (2011). Epitaxially grown crystalline silicon thin-film solar cells reaching $16.5 \%$ efficiency with basic cell process. Thin Solid Films. [Online]. vol. 519, no. 10, pp. 3288-3290. Available: http://www.sciencedirect.com/science/article /pii/S0040609010017992

[41] R. A. Rao, L. Mathew, S. Saha, S. Smith, D. Sarkar, R. Garcia, R. Stout, A. Gurmu, E. Onyegam, D. Ahn, D. Xu, D. Jawarani, J. Fossum, and S. Banerjee, "A novel low cost $25 \mu \mathrm{m}$ thin exfoliated monocrystalline $\mathrm{Si}$ solar cell technology," in Proc. IEEE Photovoltaic Spec. Conf., Jun. 2011, pp. 001 504-001507.

[42] J. H. Petermann, D. Zielke, J. Schmidt, F. Haase, E. G. Rojas, and R. Brendel. (2012). 19\%-efficient and $43 \mu \mathrm{m}$-thick crystalline $\mathrm{Si}$ solar cell from layer transfer using porous silicon. Prog. Photovoltaics: Res. Appl. [Online]. vol. 20, no. 1, pp. 1-5, 2012. Available: http://dx.doi.org/10.1002/pip.1129
Emily D. Kosten received the Ph.D. degree in physics from the California Institute of Technology (CalTech), Pasadena, CA, USA, where she received the Resnick Sustainability Institute Graduate Fellowship.

Her Ph.D. research interests in the Atwater group include optical design and the thermodynamic limits of solar cell efficiency. After completing her studies at Caltech, she will join the technical staff of the Lincoln Laboratory, Massachusetts Institute of Technology, Lexington, MA, USA.

Bonna K. Newman (M'14) received the Ph.D. degree in physics from the Massachusetts Institute of Technology, (MIT) Cambridge, MA, USA, in 2008.

She was a Clare Boothe Luce Postdoctoral Fellow with the Laboratory for Photovoltaics, MIT, researching defect engineering in materials for photovoltaics from 2008 until 2010. From 2011 to 2013, she was a Senior Member of the Technical Staff with Twin Creeks Technologies, San Jose, CA, USA, developing HIT cells on c-Si substrates that were less than 20- $\mu$ m thick. She joined the Center for Nanophotonics, FOM Institute AMOLF, Amsterdam, Netherlands, in 2013 to study the application of nanophotonic concepts to solar cell devices and recently joined the research staff at ECN Solar Energy, Petten, the Netherlands. Her research interests include light capture on thin Si substrates, nanostructured photovoltaics, and advanced device concepts with industrial viability.

John V. Lloyd received the B.S. and M.S. degrees in materials science and engineering from Drexel University, Philadelphia, PA, USA, in 2010. He is currently working toward the Ph.D. degree with the California Institute of Technology, Pasadena, CA, USA.

His current research interests include optical strategies for enhancing photovoltaic energy conversion efficiencies.

Albert Polman received the Ph.D. degree from the University of Utrecht, Utrecht, the Netherlands, in 1989.

He was a Postdoctoral Researcher with AT\&T Bell Laboratories until 1991. $\mathrm{He}$ is the Scientific Group Leader with the FOM Institute AMOLF, Amsterdam, the Netherlands, where he heads the Program Light management in new photovoltaic materials. He is a Professor of Photonic materials for photovoltaics with the University of Amsterdam. He became group leader at AMOLF, where he also served as director during 2006-2013. His research interests include nanophotonics, with special emphasis on light management in solar cells and optical metamaterials. He has published more than 250 papers and has 10 patent applications. His group has developed novel generic concepts of light coupling, light trapping, and current collection using nanostructured metasurfaces that can be applied to nearly all types of solar cells.

Harry A. Atwater (M'13) received the B.S., M.S., and Ph.D. degrees from the Massachusetts Institute of Technology, Cambridge, MA, USA, in 1981, 1983, and 1987, respectively.

He held the IBM Postdoctoral Fellowship at Harvard University, Cambridge, from 1987 to 1988, and has been a member of the faculty with the California Institute of Technology, Pasadena, CA, USA, since 1988. He is the Howard Hughes Professor of applied physics and materials science with the California Institute of Technology. He currently serves as a Director of the DOE Energy Frontier Research Center on Light-Material Interactions in Solar Energy Conversions and is also Director of the Resnick Sustainability Institute, which is Caltechs largest endowed research program. His scientific interests have two themes: photovoltaics and solar energy, as well as plasmonics and optical metamaterials. His group has created new high-efficiency solar cell designs and have developed principles for light trapping. He is an early pioneer in nanophotonics and plasmonics; he coined the term plasmonics in 2001. He has authored or coauthored more than 400 publications cited in aggregate more than 20000 times, and his group's advances in the solar energy and plasmonics field have been reported in Scientific American, Science, Nature Materials, Nature Photonics, and Advanced Materials. He is the founding Editor-in-Chief of ACS Photonics and serves as Associate Editor for the IEEE JOURNAL OF PHOTOVOLTAICS. In 2006, he founded the Gordon Research Conference on Plasmonics, which he served as chair in 2008. He is co-Founder and Chief Technical Advisor for Alta Devices, which is a venture-backed company in Santa Clara, CA, currently developing high-efficiency/low-cost GaAs photovoltaics technology, and of Caelux Corporation, which is a venture-backed photovoltaics company in Pasadena. 